\title{
A Mild Phenotype of Mitochondrial DNA Depletion Syndrome Type 13 with a Novel FBXL4 Variant
}

\author{
Ummuhan Oncul Engin Kose Fatma Tuba Eminoglu \\ Department of Pediatric Metabolism, Faculty of Medicine, Ankara University, Ankara, Turkey
}

\section{Established Facts}

- mtDNA depletion syndrome 13 (MTDPS13) is an encephalomyopathic rare genetic disorder caused by FBXL4 variants.

- Developmental delay, generalized hypotonia, feeding difficulties, and failure to thrive as well as hyperlactatemia are the most common features of MTDPS13.

- To date, 53 pathogenic FBXL4 variants and 100 cases have been described in the literature.

\section{Novel Insights}

- While most of the patients with FBLX4 gene mutations have severe clinical manifestation and die at a very young age, clinical progress of our 4.5 -year-old patient was milder than previously reported.

- Cardiac involvement commonly presents with nonprogressive cardiomyopathy, but our patient's cardiomyopathy was mildly progressive.

- In this case, a novel homozygous variant c.486T $>\mathrm{G}\left(\mathrm{p} . \mathrm{Y} 162^{*}\right)$ in the FBXL4 gene (NM_012160.4) was identified.

\section{Keywords}

Encephalopathy · FBXL4 - Mitochondrial DNA depletion · mtDNA depletion syndrome · Myopathy, MTDPS13

\footnotetext{
Abstract

Mitochondrial DNA depletion syndromes (MDDS) are a group of rare genetic disorders caused by defects in multiple genes involved in mitochondrial DNA maintenance. Among these, FBXL4 gene variants result in encephalomyopathic mtDNA depletion syndrome 13 (MTDPS13), which common-
}

ly presents as a combination of failure to thrive, neurodevelopmental delays, encephalopathy, hypotonia, a pattern of mild facial dysmorphisms, and persistent lactic acidosis. To date, 53 pathogenic FBXL4 variants and 100 cases have been described in the literature. In the present case report, we report on a 4.5-year-old boy with MTDPS13 and a novel variant. The patient had a history of antenatal hydrocephalus, severe developmental delay and mental motor retardation with psychomotor delay, severe hypotonia, mild left ventricular hypertrophic cardiomyopathy, mild facial dysmorphism, and elevated lactate levels. Symptoms suggested mitochon-
Correspondence to:

Ummuhan Oncul, onculummuhan @gmail.com 
drial myopathy; subsequently, whole-exome sequencing was performed and a novel homozygous variant FBXL4 (NM_012160.4): c.486T>G (p.Tyr162Ter) was identified. While most of the patients with $F B L X 4$ gene mutation have severe clinical manifestation and die at a very young age, clinical progress of our case was milder than previously reported. MDDS are very rare and can present with many different clinical signs and symptoms. In this report, we identified a novel pathogenic variant in the $F B X L 4$ gene. This report shows that patients with $F B L X 4$ gene mutations may present with a milder clinical phenotype than previously reported.

(c) 2021 S. Karger AG, Basel

\section{Introduction}

Mitochondria are dynamic organelles that undergo fusion, fission, movement, and autophagy. Mitochondrial dynamic processes are mediated by several nuclear-encoded proteins that function in mitochondrial fusion and fission. Pathogenic variants in several nuclear genes that are known to be crucial for mtDNA maintenance have been linked to mtDNA maintenance defects, including genes encoding the proteins involved in mitochondrial fusion (OPA1, MFN2, and FBXL4) [El-Hattab et al., 2017a]. It is believed that F-box and the leucine-rich repeat 4 (FBXL4; encoded by the FBXL4 gene) protein play a role in the mitochondrial fusion process, either by acting as a fusion protein or through their interaction with and regulation of other mitochondrial fusion proteins [Bonnen et al., 2013; Gai et al., 2013; El-Hattab et al., 2017b].

Encephalomyopathic mtDNA depletion syndrome 13 (MTDPS13; OMIM \#615471) is an extremely rare autosomal recessive disorder caused by biallelic mutations in the FBXL4 gene (MIM 605654), with an estimated prevalence of $1 / 100,000-400,000$. The neonatal period is the typical time for presentation, with $75 \%$ of the affected individuals showing symptoms by the age of 3 months [Suarez-Rivero et al., 2016]. FBXL4-related encephalomyopathy is a multisystem disease that affects mainly the central nervous system, heart, and liver. Lactic acidemia, developmental delay, and generalized hypotonia are universal, and feeding difficulties and growth failure are common features. Various distinctive facial features have been identified in some affected individuals, including prominent forehead, synophrys, thick eyebrows, long eyelashes, epicanthus, short palpebral fissures, hypertelorism, broad and depressed nasal bridge, long and smooth philtrum, thin upper lip vermilion, and low-set ears [El-Hattab et al., 2017b]. About half of those affected have microcephaly and hyperammonemia. Recently, gastrointestinal manifestations have been reported in 2 cases [Köse et al., 2020; Nardi et al., 2020]. The prognosis is variable, with death being reported in $30 \%$ of the cases at a mean age of 3 years, and a median of 2 years.

Here, we report on a 4.5-year-old Turkish boy with milder clinical progress of encephalomyopathic MTDPS13 and with a novel nonsense FBXL4 mutation (NM_012160.4): c.486T>G (p.Tyr162Ter) was detected.

\section{Case Report}

The patient, a 4.5-year-old boy - the third child of consanguineous parents (Fig. 1a), was born at 37 weeks of gestation via Csection, with antenatal hydrocephaly and polyhydramnios. His weight at birth was 2,250 g (10th-50th percentile), and his occipital-frontal circumference (OFC) was $34.5 \mathrm{~cm}$ (50th-90th percentile), according to Fenton growth chart. He was admitted to the neonatal intensive care unit for 45 days due to hydrocephaly, vomiting and poor oral feeding, where orogastric tube feeding was initiated.

At the age of 7 months, the percutaneous gastric feeding tube was replaced, and the patient underwent surgery for unilateral undescended testis and bilateral inguinal hernia at the age of 8 months. At 11 months of age, the patient was evaluated for metabolic disease due to severe hypotonia, failure to thrive, developmental delay, and hyperlactatemia. His height was $62 \mathrm{~cm}(<3 \mathrm{rd}$ percentile), weight $6 \mathrm{~kg}(<3 \mathrm{rd}$ percentile) and OFC $42.5 \mathrm{~cm}(<3 \mathrm{rd}$ percentile), according to the WHO child growth standards. He had evident dysmorphic features, including a prominent and broad forehead and a metopic ridge, thick eyebrows, short palpebral fissures, epicanthic folds, a broad nasal bridge, smooth philtrum, and a small chin (Fig. 1b). Neurological examination showed hypotonia, upbeat nystagmus and strabismus. Other system examinations were unremarkable.

Laboratory examination revealed mild renal tubular acidosis and hypercalciuria. The acylcarnitine profile analysis was normal. His plasma ammonia level was slightly elevated with $52 \mu \mathrm{mol} / \mathrm{L}$, reference range (RR) 11-35, while creatine kinase was $143 \mathrm{U} / \mathrm{L}$ (RR 32-294). Plasma amino acid level analysis revealed elevated alanine level of $1,233 \mathrm{mmol} / \mathrm{L}$ (RR 74-400), consistent with hyperlactatemia. Plasma lactate levels were $50 \mathrm{mg} / \mathrm{dL}$ (RR 10-14), and at follow-up, hyperlactatemia was persistent (mean level: $72.7 \mathrm{mg} /$ $\mathrm{dL}$, minimum: $36.9 \mathrm{mg} / \mathrm{dL}$, maximum: $112 \mathrm{mg} / \mathrm{dL}$ ). Urine organic acid analysis revealed elevated levels of metabolites from the citric acid cycle, including lactic acid and fumaric acid, compatible with mitochondrial dysfunction.

At the age of 1 year, his EEG was abnormal, with bilateral frontal high amplitude slow waves, indicative of moderate-severe cerebral dysfunction. No epileptic activity was noted. An elongated visual evoked potential latency was detected (right 138, left 142 $\mathrm{msec}$ ). Brainstem auditory evoked potential test was normal, while abdominal ultrasonography revealed nephrocalcinosis. Mild left ventricular hypertrophic cardiomyopathy was revealed on echocardiography. 


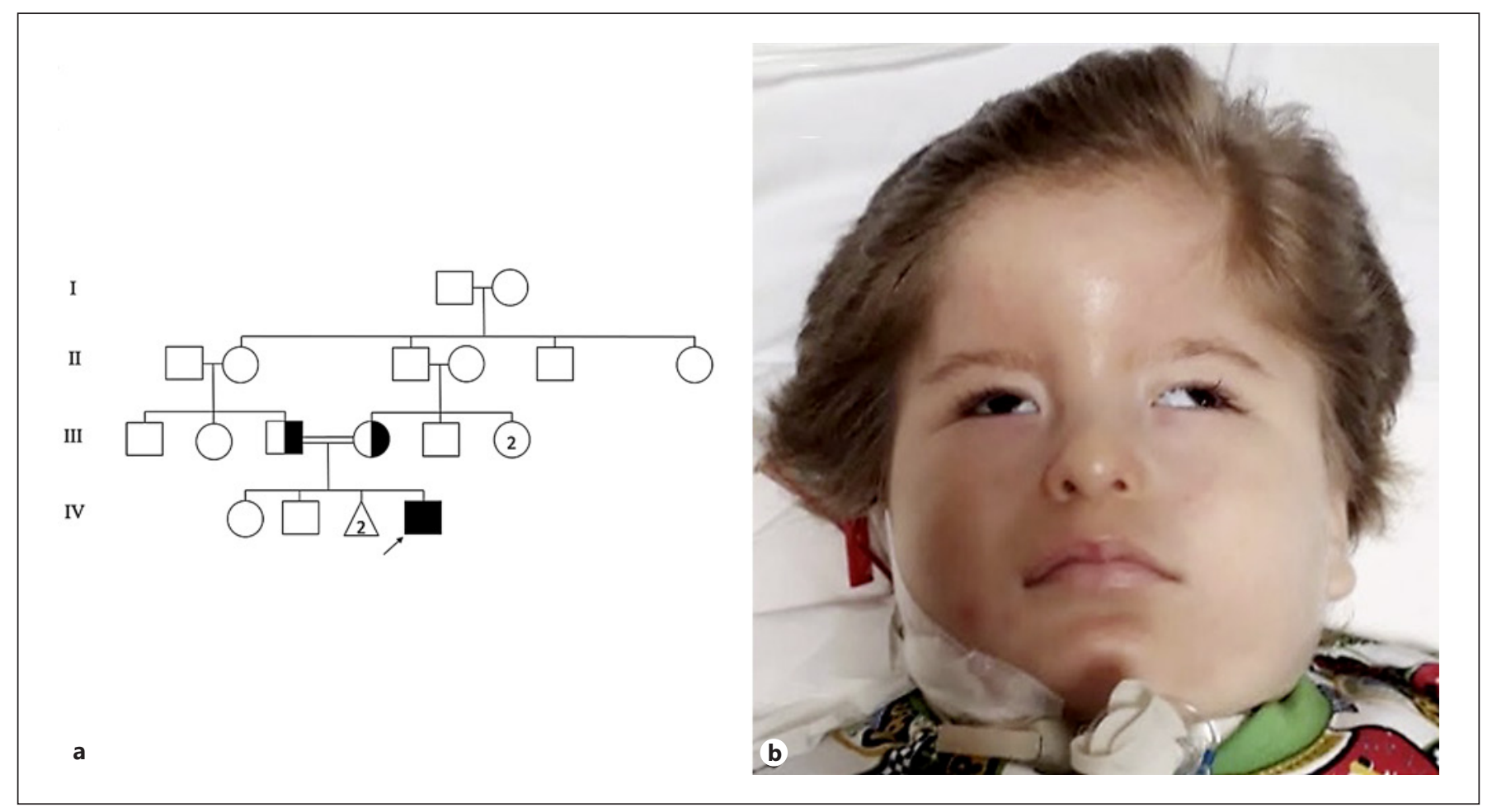

Fig. 1. a Pedigree of the patient. b Facial frontal view of our patient at age 4.5 years showing prominent and broad forehead and a metopic ridge, thick eyebrows, short palpebral fissures, epicanthic folds, a broad nasal bridge, smooth philtrum, a small chin, and strabismus.

At 1 year of age, cranial MRI examination revealed cystic encephalomalacia in the frontal lobe, thin corpus callosum, large ventricles, and cerebral atrophy. The T2-weighted image showed generalized volume loss, mostly in white matter; white matter hyperintense signal, as well as secondary enlargement of the ventricular system and external subarachnoidal spaces. Diffusion-weighted imaging revealed restricted diffusion involving periventricular white matter and focal necrotic lesions in the lentiform nucleus (bilateral). Cerebral magnetic resonance spectroscopy showed an inverted doublet lactate peak in the voxel corresponding to the brain parenchyma, and also in areas without focal lesions (Fig. 2).

All of these clinical and laboratory findings were compatible with mitochondrial disorders, so whole-exome sequencing analysis was performed using Novaseq platform (Illumina, San Diego, CA, USA) after the standard enrichment protocol of Trusight Enrichment kit (Illumina). Base calling and quality control study were performed with Real-Time Analysis software (Illumina). Sequence was compared with hg19/GRCh37 standard sequence with Genome Multitool software. Filtering by population frequencies and in silico prediction tool parameters was done, and high-risk variants were evaluated by their clinical relation to our patient. A novel homozygous nonsense c.486T $>\mathrm{G}\left(\mathrm{p} . \mathrm{Y} 162^{*}\right.$ ) variant in the FBXL4 gene was interpreted as the likely cause of the disease, as biallelic mutations in this gene were known to cause encephalomyopathic MTDPS13, and the patient's phenotype showed extensive overlap with this disease (Fig. 3). The heterozygous p.Tyr162* mutation was identified in the patient's parents.
In clinical follow-up, coenzyme Q10 (300 mg/day), biotin (5 mg/day), riboflavin (100 mg/day) and levocarnitine $(50 \mathrm{mg} / \mathrm{kg} /$ day) treatments were initiated at 1.5 -years, but no significant clinical improvement was achieved. At the age of 4.5 years, mildly progressive cardiomyopathy was revealed. At the same age, hypotonia, significant head lag, and global developmental delay were evident. He was not able to hold his head, make eye contact, react to voices and babble.

\section{Discussion}

In this study, we report a novel nonsense variant in the FBXL4 gene, c.486T>G (p.Y162*) (NM_012160.4), associated with encephalomyopathic MTDPS13 in a 4.5-yearold Turkish boy. To the best of our knowledge, 53 variants in the FBXL4 gene have been reported to date in 100 affected individuals, and our report is thus the 54th variant to be identified in literature. FBXL4 variants appear to be pan-ethnic, and the majority are missense $(26 / 53$; $49 \%)$ and nonsense mutations $(11 / 53 ; 21 \%)$. Only a few variants are frameshift $(9 / 53 ; 17 \%)$ or splice site mutations $(7 / 53 ; 13 \%)$, although more genetic reports are needed to better determinate the genotype-phenotype re- 

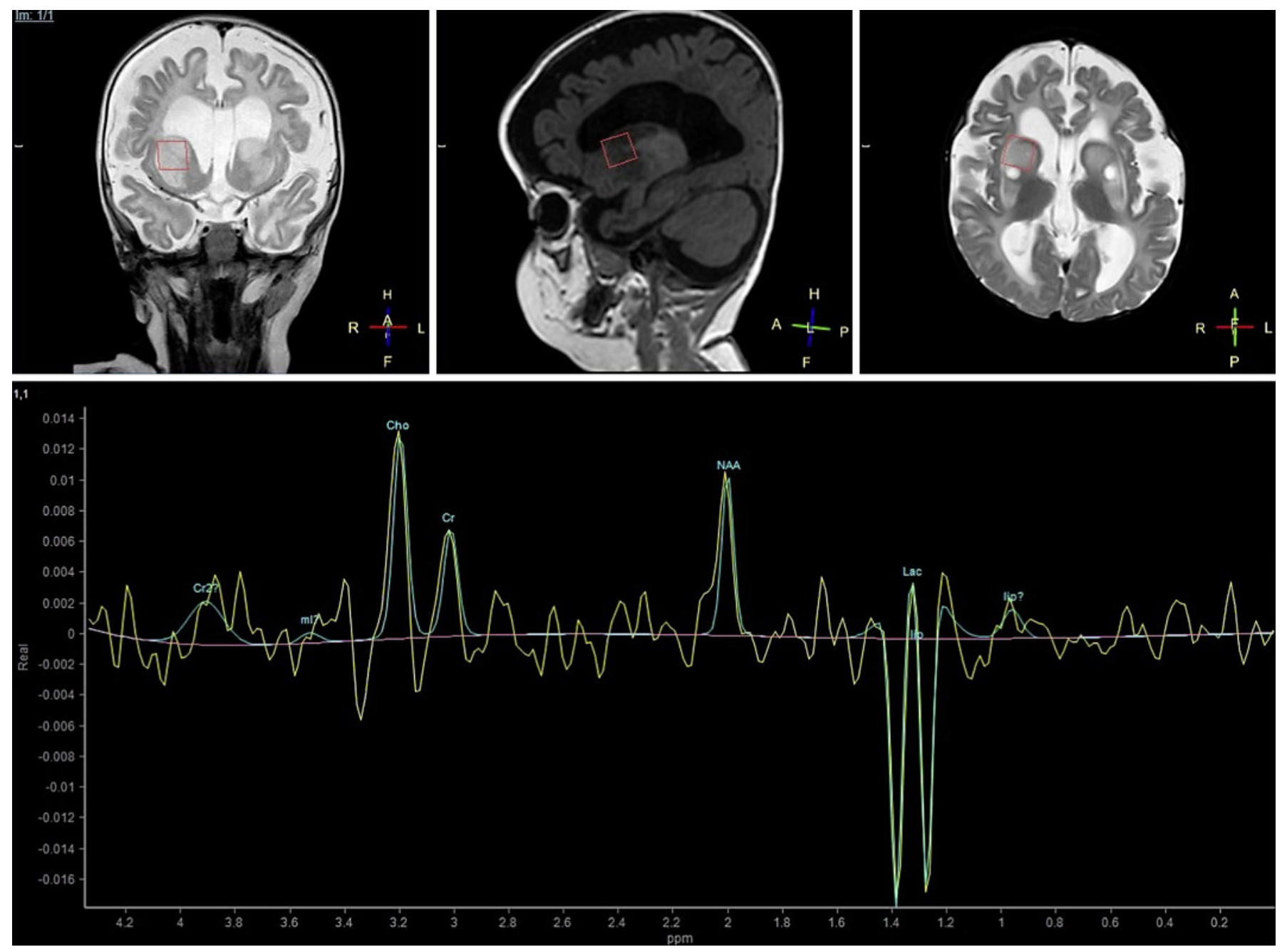

Fig. 2. Brain magnetic resonance spectroscopic imaging findings of our patient at the age of 1 year was obtained at intermediate echo-time by single-voxel $1 \mathrm{H}$ magnetic resonance spectroscopy of the right basal ganglia (red square). An inverted doublet lactate peak at $1.3 \mathrm{ppm}$ was defined.

lationship. Based on the observed pattern of clinical features in previous reports, we suggest that mutations in the FBXL4 gene be considered when children exhibit very early (neonatal in some cases) onset of lactic acidosis, profound muscular hypotonia and failure to thrive, often associated with characteristic facial features (thick eyebrows, short, upslanting palpebral fissures with epicanthus, broad nasal bridge, bulbous nasal tip, everted lower lip, and smooth and long philtrum) and feeding difficulties. While multiorgan involvement is a common finding in mitochondrial disease, the presence of a distinct dysmorphic phenotype is rare [Gai et al., 2013; Barøy et al., 2016; Dai et al., 2017]. The most common presentation in our patient and those in previously published reports, however, is congenital lactic acidosis and typical facial dysmorphisms, which appears to be a hallmark of FBXL4 defects. The prevalence of FBXL4 defects in patients suspected of having a mitochondrial disorder is approximately $0.7 \%$ [Dai et al., 2017]. Particularly when the patient presents with congenital lactic acidosis and typical facial dysmorphisms, patient should be carefully evaluated with mitochondrial disease gene panels.

In particular, the age at onset of symptoms in studies to date have been remarkably different, ranging from immediately intrauterine to 3 months [Huemer et al., 2015; van Rij et al., 2016]. Only a few individuals have been reported with symptoms after the age 6 months, although one female patient described was 13 years old [Pronicka et al., 2016]. Our patient's symptoms started in the intrauterine period, manifesting primarily with nonspecific 


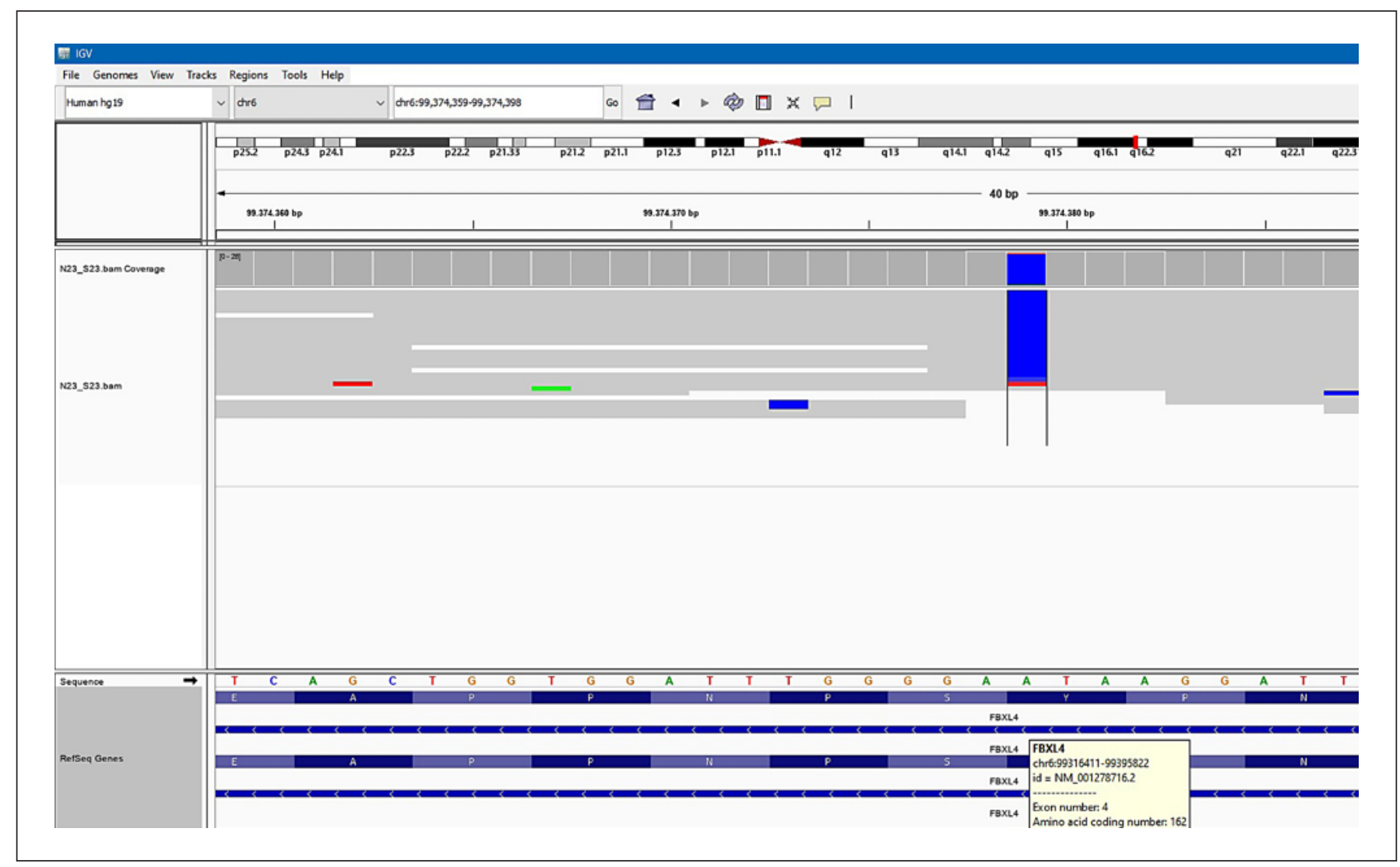

Fig. 3. Screenshot of a novel homozygote FBXL4 (NM_012160.4) c.486T>G mutation analysis by IGV 2.3 (Broad Institute) software. The sequence is represented by colored letters with adenine in green, cytosine in blue, guanine in yellow, and thymine in red (A, C, G, T).

symptoms such as growth failure, feeding difficulty, hypotonia, and global developmental delay. Many patients are critically ill at the time of the initial presentation, and may die at a very young age [El-Hattab et al., 2017b]. In the survivors, the initial pattern persists, and development, motor and cognitive abilities become severely impaired as they grow older. Cardiac manifestations of MTDPS13 include cardiomyopathy, congenital heart malformations, arrhythmias, and pulmonary hypertension. Cardiomyopathy, typically hypertrophic, is the most common cardiac manifestation of MTDPS13 and may be evident at initial presentation or may evolve within the course of the disease [Almannai et al., 2017]. In our patient, we detected a mild cardiomyopathy at 12 months of age.

Other organs are rarely affected, although gastrointestinal manifestations in patients with FBXL4 mutations have recently been reported [Köse et al., 2020; Nardi et al., 2020]. The clinical course is generally progressive, or at best stable, and the quality of life of both the patients and caregivers is considerably impaired. As the disease evolved in our patient, neurocognitive impairment, commonly severe psychomotor retardation and developmental delay ,became readily apparent. We believe that more case reports of FBXL4 variants may help to better understand the clinical manifestations of the disease.

Some of the cases reported in literature had such visual symptoms as congenital cataracts, strabismus and optic nerve atrophy [Bonnen et al., 2013]. Consistently, strabismus and upbeat nystagmus were detected in our case at the age of 11 months.

There are no specific routine or basic laboratory parameters that may facilitate diagnosis, other than lactic acidosis, and in some cases, elevated creatine kinase [Huemer et al., 2015]. Neuroimaging can show nonspecific findings, such as hypomyelination, cerebral atrophy, basal ganglia abnormalities, dilated ventricles, enlarged cisterna magna, corpus callosum thinning, cerebellar atrophy, periventricular cysts, brain stem abnormalities, and arachnoid cysts. In some patients, the central gray matter structures, including the mesencephalon, show increased 
volume, and T2 signal and decreased ADC, as is the case with other mitochondrial disorders [Huemer et al., 2015].

The current medical therapies involve various vitamins, cofactors, respiratory substrates or antioxidant compounds, but are generally ineffective. As such, the treatment of mitochondrial diseases remains largely symptomatic, and does not significantly alter the course of the disease [El-Hattab et al., 2017c]. In our patient, as expected, coenzyme Q10, biotin, riboflavin and levocarnitine treatments were initiated, but no significant clinical improvement was achieved during follow-up.

In conclusion, this case report expands the phenotype of FBXL4-associated mitochondrial encephalomyopathy and suggests that this condition may present with a milder phenotype than previously reported. The identification of further patients with novel pathogenic variants in the FBXL4 gene will help associate specific mutations with the range of disease phenotypes.

\section{Acknowledgement}

We thank the patient and his family for their helpful participation in this work.

\section{Statement of Ethics}

This study was exempt from ethical approval procedures, being a case report of a single patient whose parents provided verbal consent to participate in the study, and gave written consent to have the case published. Informed consent for genetic analysis and publication of clinical reports and photographs were obtained from the patient or his parent/guardian in compliance with the national ethics regulation. There is no name or number indicating the patient's identity.

\section{Conflict of Interest Statement}

The authors have no conflicts of interest to declare.

\section{Funding Sources}

There was no funding for this study.

\section{Author Contributions}

All authors contributed equally to this study.

\section{References}

Almannai M, Dai H, El-Hattab AW, Wong LJC. FBXL4-related encephalomyopathic mitochondrial DNA depletion syndrome. GeneReviews $^{\oplus}$ [Internet]. Seattle (WA): University of Washington, Seattle; 1993-2021). 2017.

Barøy T, Pedurupillay CRJ, Bliksrud YT, Rasmussen $\mathrm{M}$, Holmgren A, Vigeland MD, et al. A novel mutation in FBXL4 in a Norwegian child with encephalomyopathic mitochondrial DNA depletion syndrome 13. Eur J Med Genet. 2016;59:342-6.

Bonnen PE, Yarham JW, Besse A, Wu P, Faqeih EA, Al-Asmari AM, et al. Mutations in FBXL4 cause mitochondrial encephalopathy and a disorder of mitochondrial DNA maintenance. Am J Hum Genet. 2013;93(3):471.

Dai H, Zhang VW, El-Hattab AW, Ficicioglu C, Shinawi M, Lines M, et al. FBXL4 defects are common in patients with congenital lactic acidemia and encephalomyopathic mitochondrial DNA depletion syndrome. Clin Genet. 2017;91(4):634-9.

El-Hattab AW, Craigen WJ, Scaglia F. Mitochondrial DNA maintenance defects. Biochim Biophys Acta Mol Basis Dis. 2017a;1863(6): 1539.
El-Hattab AW, Dai H, Almannai M, Wang J, Faqeih EA, Al Asmari A, et al. Molecular and clinical spectra of FBXL4 deficiency. Hum Mutat. 2017b;38(12):1649-59.

El-Hattab AW, Zarante AM, Almannai M, Scaglia F. Therapies for mitochondrial diseases and current clinical trials. Mol Genet Metab. 2017c;122:1-9.

Gai X, Ghezzi D, Johnson MA, Biagosch CA, Shamseldin HE, Haack TB, et al. Mutations in FBXL4, encoding a mitochondrial protein, cause early-onset mitochondrial encephalomyopathy. Am J Hum Genet. 2013;93(3): $482-95$.

Huemer M, Karall D, Schossig A, Abdenur JE, Al Jasmi F, Biagosch C, et al. Clinical, morphological, biochemical, imaging and outcome parameters in 21 individuals with mitochondrial maintenance defect related to FBXL4 mutations. J Inherit Metab Dis. 2015;38(5): 905-14.

Köse E, Köse M, Edizer S, Akışın Z, Yılmaz ZB, Şahin A, et al. Different clinical presentation in a patient with two novel pathogenic variants of the FBXL4 gene. Turk J Pediatr. 2020; 62(4):652-56.
Nardi N, Proulx F, Brunel-Guiton C, Oligny LL, Piché N, Mitchell GA, et al. Fulminant Necrotizing Enterocolitis and Multiple Organ Dysfunction in a Toddler with Mitochondrial DNA Depletion Syndrome-13. J Pediatr Intensive Care. 2020;9(1):54-9.

Pronicka E, Piekutowska-Abramczuk D, Ciara E, Trubicka J, Rokicki D, Karkucinska-Wieckowska A, et al. New perspective in diagnostics of mitochondrial disorders: Two years' experience with whole-exome sequencing at a national paediatric centre. J Transl Med. 2016; 14(1): 174 .

Suarez-Rivero JM, Villanueva-Paz M, de la CruzOjeda P, de la Mata M, Cotan D, OropesaAvila M, et al. Mitochondrial Dynamics in Mitochondrial Diseases. Diseases. 2016;5(1): 1.

van Rij MC, Jansen FA, Hellebrekers DM, Onkenhout W, Smeets HJ, Hendrickx AT, et al. Polyhydramnios and cerebellar atrophy: A prenatal presentation of mitochondrial encephalomyopathy caused by mutations in the FBXL4 gene. Clin Case Rep. 2016;4(4):425. 